\title{
The Presence of thyroid peroxidase antibodies in Graves' disease is predictive of disease duration and relapse rates
}

Wahab.F, Kearney. E, Joseph. S

Department of Diabetes and Endocrinology, Queen Elizabeth the Queen Mother Hospital, Margate, Kent.

Background

Thyroid peroxidase antibodies (TPO) and TSH receptor antibodies (TRAB) sometimes co-exist in patients with Graves disease (GD) but not always. Some studies have suggested a functional and prognostic role for TRAB but the phenotypic characteristics of the patient with positive TPO (with or without TRAB) and the influence of TPO on the clinical course of GD are not known.

\section{Objective}

We therefore set out to examine the demographic and clinical profile of patients with GD to assess for any association between TPO antibody expression (with or without TRAB) and the clinical course of the disease.

\section{Methods}

We reviewed the health records of 14 patients with GD who were being followed up in the endocrine Clinic in East Kent between 2010 and 2011. Patients were divided into TPO-ve and TPO+ve groups and demographic and clinical data collected. Data are expressed in $\%$, mean + SD and a $p$ value of 0.05 was deemed significant

\section{Results}

9 Patients were TPO+ve. They were predominantly female (89\%) and younger compared to the TPO-ve group. A positive family history was present in $60 \%$ TPO+ve patients but in none of the TPO-ve patients. TPO+ve patients were treated for longer and had $30 \%$ relapse rate within 12 months of discontinuing therapy and $20 \%$ needed radioactive iodine therapy (RAI) early. None of the TPO-ve relapsed nor required RAI. TRAB was also higher in the TPO-ve patients

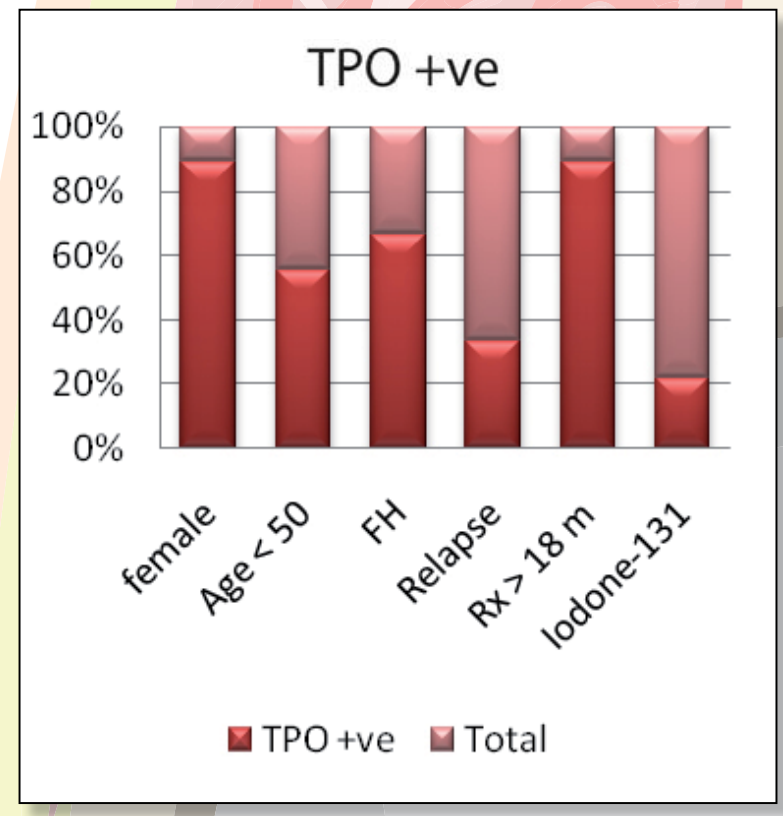

This study has demonstrated that the presence of TPO in Graves disease results in a phenotype of patients with a more aggressive disease pattern that occurs mainly in females and younger age group, takes longer to treat and has a higher relapse rate compared to TPO -ve patients. This finding has practical implications for the management of GD but larger studies will be required to confirm the findings.

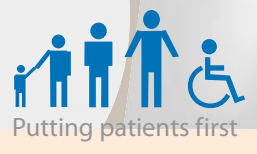

